\title{
Newton regularizations for impedance tomography: a numerical study*
}

\author{
Armin Lechleiter ${ }^{1}$ and Andreas Rieder ${ }^{2}$ \\ ${ }^{1}$ Graduiertenkolleg 1294 'Analysis, Simulation and Design of Nanotechnological Processes', \\ Universität Karlsruhe, 76128 Karlsruhe, Germany \\ ${ }^{2}$ Institut für Angewandte und Numerische Mathematik and Institut für Wissenschaftliches \\ Rechnen und Mathematische Modellbildung, Universität Karlsruhe (TH), 76128 Karlsruhe, \\ Germany \\ E-mail: armin.lechleiter@math.uni-karlsruhe.de and andreas.rieder@math.uni-karlsruhe.de
}

Received 29 May 2006, in final form 20 July 2006

Published 27 September 2006

Online at stacks.iop.org/IP/22/1967

\begin{abstract}
The inexact Newton iteration REGINN for regularizing nonlinear ill-posed problems consists of two components: the (outer) Newton iteration, stopped by a discrepancy principle, and the inner iteration, which computes the Newton update by solving approximately a linearized system. The second author proved the convergence of REGINN furnished with the conjugate gradients method as inner iteration (Rieder 2005 SIAM J. Numer. Anal. 43 604-22). Amongst others the following feature distinguishes REGINN from other Newton-like regularization schemes: the regularization level for the locally linearized systems can be adapted dynamically incorporating information on the local degree of ill-posedness gained during the iteration. Of course, the potential of this feature can be fully explored only by meaningful numerical experiments in a realistic setting. Therefore, we apply REGINN to the 2D-electrical impedance tomography problem with the complete electrode model. This inverse problem is known to be severely ill-posed. The achieved reconstructions are compared qualitatively and quantitatively with reconstructions from a one-step method which is closely related to the NOSER algorithm (Cheney et al 1990 Int. J. Imag. Syst. Technol. 2 66-75), an often used solver in impedance tomography. Our detailed numerical comparison reveals REGINN to be a competitive solver outperforming the one-step method when noise corrupts the data and/or a moderately large number of electrodes is used.
\end{abstract}

\footnotetext{
* Financial support by the Institut für Wissenschaftliches Rechnen und Mathematische Modellbildung, Universität Karlsruhe (TH), and the Mathematical Department of the University of Delaware, USA, is gratefully acknowledged. 


\section{Introduction}

Electrical impedance tomography (EIT) entails the determination of the electric conductivity distribution of an object by applying electric currents at the boundary through electrodes and measuring the resulting voltages at the boundary as well. Potential applications are, for instance, medical imaging and non-destructive testing.

Because of its promising applications and its challenging mathematics EIT attracted a vast amount of research during the last two decades, both theoretically and practically; all starting out from the pioneering work of Caldéron [11]. The nonlinearity and the severe ill-posedness of EIT remain a challenge for reconstruction algorithms nonetheless. Algorithms known to us can be categorized as

1. noniterative algorithms based on global linearization,

2. iterative solvers tackling the full nonlinear problem and

3. direct methods.

Noniterative algorithms based on global linearization can be built by stopping any iterative algorithm after the first step, a prominent example is the NOSER algorithm [13]. For the iterative inverse solvers one usually exploits Fréchet differentiability of the forward operator and uses a regularized Newton-type method. A somewhat different approach is propagated in [5] where a nonlinear multigrid method solves a Tikhonov-regularized firstorder optimality condition of an output least-squares formulation. The class of direct methods splits into two subclasses: (a) factorization methods use special singular testfunctions to characterize inclusions in a homogeneous background medium [7-10] and (b) direct methods that implement a constructive existence and uniqueness proof $[1,26,33,34]$. As far as we know both direct methods are not able to deal with finite electrode models but need to apply currents and measure the voltages along the whole boundary of the object (in mathematical terms: they need to observe the Neumann-to-Dirichlet mapping). Their use for a realistic setting is therefore limited.

Our work at hand contributes to the second class: we apply the nonlinear regularization method REGINN (REGularization based on INexact Newton iterations), developed and analysed by the second author [29, 30, 32], to the 2D-EIT problem with the complete electrode model. The most delicate part of any Newton-like regularization is the stable computation of the Newton step from the locally linearized system. As the degree of ill-posedness of the locally linearized system may change dramatically during the Newton iteration, a careful selection of the level of regularization of the linear system is indispensable. Surprisingly, this is not the case for most Newton methods, see, e.g., [4, 24]. Also the nonlinear multigrid method from [5] works with a priori regularization parameters on the intermediate grids. In contrast, REGINN selects the level of regularization of the locally linearized system incorporating information on the local degree of ill-posedness gained during the iteration. To this end, we monitor the numerical effort for computing the Newton steps. An increase (decrease) of this numerical effort indicates an increase (decrease) of the local degree of ill-posedness. Accordingly, the magnitude of the regularization for the following linearized system is adjusted.

To put REGINN in perspective we compare it with a one-step solver being akin to the NOSER algorithm. In spite of its simplicity the one-step solver delivers reconstructions of an astonishing quality. In particular, when only a small number of electrodes is used, that is, the data contain only little information on the conductivity, the one-step solver is hard to beat. Nevertheless, the REGINN reconstructions contain less noise and appear more focused with a higher contrast. They are also quantitatively better than the reconstructions by the one-step solver. 


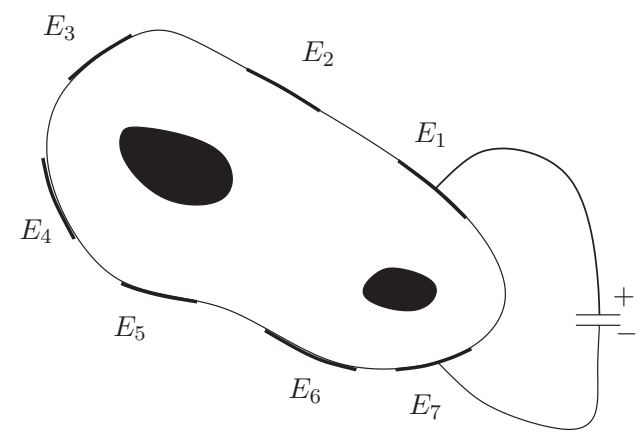

Figure 1. The experimental set-up of an EIT tomography system with seven electrodes. The body under investigation contains two inclusions.

We start our paper in the next section by introducing the mathematical model for EIT we work with. For the discretization of the governing elliptic equation, we rely on finite elements as we show in section 3. Section 4 is devoted to the Fréchet differentiability of the EIT operator allowing us to tackle the inverse problem by Newton-like solvers in section 5. Here, we also report the numerical experiments with the NOSER-like one-step solver (section 5.1). Next we present REGINN with the conjugate gradients method as inner iteration (section 6) followed by numerical experiments and a qualitative comparison of both solvers. The quantitative comparison together with our conclusions is content of the final section. The paper ends with the appendix where we explain how to compute efficiently the Fréchet derivative of the discretized EIT operator.

\section{The complete electrode model}

In this section, we give a brief account on the mathematical model for EIT.

Assume that $p$ electrodes have been fixed around the surface of the object, for instance around a human chest (see figure 1). Current is applied to some subset of these electrodes and the resulting voltages at all $p$ electrodes are measured. This procedure, called the EIT experiment, is repeated several times with different electrodes until a sufficient amount of data has been gained. The inverse problem of EIT is then to reconstruct the inner structure of the investigated object using this data set. Clearly, the EIT problem can be solved only if the inner structure consists of areas with substantially different conductivities. In medical imaging this prerequisite is often fulfilled, see Cheney, Isaacson and Newell [12, 13].

In the corresponding forward problem one wants to find the electric potential in the interior of the object and at the electrodes, given some applied current. If we assume that the object under consideration does not contain any current source in its interior and that the frequency of the current is small enough then a scaling analysis [12] shows that Maxwell's equations describing the electromagnetic fields inside the object reduce to the elliptic equation

$$
\nabla \cdot(\sigma \nabla u)=0 \quad \text { in } B
$$

where $\sigma$ denotes the electric conductivity in the object $B$ and $u$ denotes the voltage potential. We assume in the following that $\sigma$ is a bounded positive scalar function in the closure $\bar{B}$ of $B$. Moreover, $\sigma$ is assumed to be Lipschitz continuous in $\bar{B}$ with possible jump discontinuities in $B$. Thus, a trace $\left.\sigma\right|_{\partial B}$ is meaningfully defined. We denote the class of admissible conductivities 
by

$$
\begin{gathered}
\mathcal{A}:=\left\{\sigma \in L^{\infty}(B) \mid \sigma \geqslant \sigma_{0}>0, \text { there are }\left(B_{j}\right)_{j=1}^{m}: B_{j} \Subset B, B_{j}\right. \text { open, } \\
\left.\left.\sigma\right|_{B_{j}} \in W^{0,1}\left(B_{j}\right), \cup_{j} \bar{B}_{j}=\bar{B}\right\} .
\end{gathered}
$$

In the case of real conductivities, several uniqueness results for the inverse problem have been proved, see for instance [2, 27, 35-37].

A careful modelling of the electrodes turns out to be of highest importance when comparing the predictions of the resulting mathematical models with experimental data $[14,15]$. The complete electrode model $[12,14]$, nowadays the standard model for medical applications, takes into account the following three physical properties of the EIT experiment.

First, the electrodes are a discrete set. Let us denote by $E_{1}, \ldots, E_{p}$ the $p$ electrodes, each $E_{j}$ is considered to be an open subset of the boundary $\partial B$ with positive surface measure. We assume furthermore that the $E_{j}$ 's are connected and separated, i.e., $\operatorname{dist}\left(E_{k}, E_{j}\right)>0$ for $k \neq j$. Let $I_{j} \in \mathbb{R}$ be the current applied to $E_{j}$ and define $I=\left(I_{1}, \ldots, I_{p}\right)^{\top}$. Due to the principle of conservation of charge we require that $\sum I_{j}=0$. The vector $I$ is called current pattern or current vector. For convenience, let us denote the space of current patterns of length $p$ by

$$
\mathbb{R}_{\diamond}^{p}:=\left\{I \in \mathbb{R}^{p} \mid \sum_{j} I_{j}=0\right\} .
$$

Second, we model the electrode $E_{j}$ to be a perfect conductor, that is, we assume that the potential along this electrode is constant: $\left.u\right|_{E_{j}}=$ const. This is the so-called shunting effect. To ease the notational burden, let

$$
\left.u\right|_{E_{j}}=: U_{j} \quad \text { for } \quad j=1, \ldots, p .
$$

Note that $U:=\left(U_{1}, \ldots, U_{p}\right)^{\top}$ is measured in the EIT experiment. To end up with a wellposed problem we need an additional boundary condition. As we model the electrodes as perfect conductors, the current sent to these electrodes is applied completely to $B$. This implies that the total flux over $E_{j}$ equals $I_{j}$ :

$$
\int_{E_{j}} \mathcal{B}_{\nu} u \mathrm{~d} S=I_{j} \quad \text { for } \quad j=1, \ldots, p, \quad \text { where } \quad \mathcal{B}_{\nu} u:=\sigma \nabla u \cdot v
$$

is the conormal derivative and $v$ denotes the outer unit normal to $B$.

Third, the complete electrode model includes the effect of contact impedance at the electrodes: when EIT is used in a medical context, a thin layer with high resistivity forms at the boundary between the electrodes and the skin due to dermal moisture. We incorporate this effect by introducing constants $z_{j}, j=1, \ldots, p$, which denote the positive resistivity of the contact layer at electrode $E_{j}$. According to Ohm's law the potential $u$ at $E_{j}$ drops by $z_{j} \mathcal{B}_{v} u$.

Hence, the complete electrode model gives rise to the following (weak) formulation of the forward problem: given a current vector $I=\left(I_{1}, \ldots, I_{p}\right) \in \mathbb{R}_{\diamond}^{p}$, a conductivity $\sigma \in \mathcal{A}$ and positive contact impedances $z_{1}, \ldots, z_{p}$ find a potential $u \in H^{1}(B)$ and a set of electrode voltages $U \in \mathbb{R}_{\diamond}^{p}$ that satisfy

$$
\begin{array}{ll}
\nabla \cdot(\sigma \nabla u)=0 & \text { in } B, \\
u+z_{j} \mathcal{B}_{\nu} u=U_{j} & \text { on } E_{j}, \\
\int_{E_{j}} \mathcal{B}_{\nu} u \mathrm{~d} S=I_{j} & \text { for } \quad j=1, \ldots p, \\
\mathcal{B}_{\nu} u=0 & \text { on } \partial B \backslash \cup_{j=1}^{p} \bar{E}_{j} .
\end{array}
$$


The condition $U \in \mathbb{R}_{\diamond}^{p}$, i.e., $\sum_{j=1}^{p} U_{j}=0$, can be interpreted as a grounding of the potential. Indeed, without this condition the above problem would not be unique. According to [14], the accuracy of the model matches the measurement precision of the experiment. Note that we assume in the following that the contact impedances $z_{j}$ are known and not part of the inverse problem.

Existence and uniqueness of a solution $(u, U) \in H^{1}(B) \oplus \mathbb{R}_{\diamond}^{p}$ can been shown using the Lax-Milgram lemma. Indeed, in [14] it is shown that $(u, U)$ fulfils (2.2)-(2.5) if and only if

$$
b((u, U),(v, V))=f(v, V)
$$

for all $(v, V) \in H^{1}(B) \oplus \mathbb{R}_{\diamond}^{p}$ where the strictly elliptic bilinear form $b$ is defined by

$$
b((u, U),(v, V)):=\int_{B} \sigma \nabla u \nabla v \mathrm{~d} x+\sum_{j=1}^{p} \frac{1}{z_{j}} \int_{E_{j}}\left(u-U_{j}\right)\left(v-V_{j}\right) \mathrm{d} S,
$$

and $f(v, V):=\sum_{j=1}^{p} I_{j} V_{j}$ for $(v, V) \in H^{1}(B) \oplus \mathbb{R}_{\diamond}^{p}$.

\section{Discretization by FEM}

Since the solution $(u, U)$ of the forward problem cannot be computed analytically we use the finite element method (FEM) to find an approximate solution. Following the usual procedure of FEM we use a triangulation $\mathcal{T}=\left\{T_{1}, \ldots, T_{|\mathcal{T}|}\right\}$ of the domain $B$ and define the finitedimensional subspace $H_{h}$ of $H^{1}(B)$ to be the set of continuous functions in $H^{1}(B)$ that are piecewise linear on each triangle of $\mathcal{T}$. Suppose that the triangulation $\mathcal{T}$ consists of $\ell$ nodes. Then we denote by $\phi_{k}$ the hat function that takes the value 1 at node $k$ and vanishes at all the other nodes. Any element $u_{h}$ in $H_{h}$ is represented by

$$
u_{h}=\sum_{k=1}^{\ell} \alpha_{k} \phi_{k} \quad \text { for } \quad \alpha_{k} \in \mathbb{R}
$$

For notational reasons we identify $u_{h}$ with its coordinates in the basis $\left\{\phi_{k}\right\}$ and write $u_{h}=$ $\left(\alpha_{1}, \ldots, \alpha_{\ell}\right)$. Finally, we still denote the voltages at the $p$ electrodes by $U=\left(U_{1}, \ldots, U_{p}\right) \in$ $\mathbb{R}_{\diamond}^{p}$.

Testing $u_{h}$ and $U$ in (2.6) against $v=\phi_{i}$ and $V=0$ yields

$$
\sum_{k=1}^{\ell} \alpha_{k} \int_{B} \sigma \nabla \phi_{k} \nabla \phi_{i} \mathrm{~d} x+\sum_{j=1}^{p} \frac{1}{z_{j}} \int_{E_{j}}\left(\sum_{k=1}^{\ell} \alpha_{k} \phi_{k}-U_{j}\right) \phi_{i} \mathrm{~d} S=0,
$$

for $i=1, \ldots, \ell$. The discrete system (3.1) gives rise to a matrix-vector equation in the following way: let $A \in \mathbb{R}^{\ell \times \ell}$ be the admittance matrix with entries

$$
A_{i, k}=\int_{B} \sigma \nabla \phi_{k} \nabla \phi_{i} \mathrm{~d} x+\sum_{j=1}^{p} \frac{1}{z_{j}} \int_{E_{j}} \phi_{k} \phi_{i} \mathrm{~d} S
$$

Furthermore, let $B \in \mathbb{R}^{\ell \times p}$ be the matrix defined by

$$
B_{i, j}=-\frac{1}{z_{j}} \int_{E_{j}} \phi_{i} \mathrm{~d} S .
$$

With these definitions equation (3.1) can be rewritten as $A u_{h}+B U=0$. Until now we have ignored the boundary conditions for $u_{h}$ arising from the complete model. Testing $u_{h}$ and $U$ in (2.6) now against $v=0$ and $V^{i}=\left(\delta_{i, k}\right)_{k=1}^{p}$ we find that

$$
\frac{1}{z_{i}} \int_{E_{i}}\left(U_{i}-\sum_{k=1}^{\ell} \alpha_{k} \phi_{k}\right) \mathrm{d} S=I_{i}
$$


or equivalently that

$$
U_{i} \frac{\left|E_{i}\right|}{z_{i}}-\sum_{k=1}^{\ell} \frac{\alpha_{k}}{z_{i}} \int_{E_{i}} \phi_{k} \mathrm{~d} S=I_{i}, \quad \text { for } \quad i=1, \ldots, \ell .
$$

Introducing the diagonal matrix $D \in \mathbb{R}^{p \times p}$,

$$
D_{i, i}=\frac{1}{z_{i}} \int_{E_{i}} \mathrm{~d} S=\frac{\left|E_{i}\right|}{z_{i}}
$$

we may write (3.2) as $B^{\top} u_{h}+D U=I$. Finally, we end up with the linear system

$$
\left(\begin{array}{ll}
A & B \\
B^{\top} & D
\end{array}\right)\left(\begin{array}{l}
u_{h} \\
U
\end{array}\right)=\left(\begin{array}{l}
0 \\
I
\end{array}\right)
$$

for computing the FEM solution of the forward problem. The above system has to be augmented to guarantee the grounding condition $\sum_{j} U_{j}=0$. An easy way to include this constraint is to solve

$$
\left(\begin{array}{ll}
A & B \\
B^{\top} & D \\
0 & \mathbb{1}
\end{array}\right)\left(\begin{array}{l}
u_{h} \\
U
\end{array}\right)=\left(\begin{array}{l}
0 \\
I \\
0
\end{array}\right)
$$

where $\mathbb{1} \in \mathbb{R}^{1 \times p}$ is the row vector $(1, \ldots, 1)$. This straightforward approach, however, destroys symmetry and positive definiteness of (3.3). Kaipio et al [21] suggest a more sophisticated way how to augment system (3.3) respecting its favourable structure.

A priori error estimates for the FEM solution $u_{h}$ are difficult to obtain since the solution $u$ of the complete model does not belong to $H^{2}(B)$. This is due to the possible jumps of $\sigma \in \mathcal{A}$ and because the Neumann boundary values $\mathcal{B}_{\nu} u$ do only belong to $H^{s}(\partial B)$ for $s<1 / 2$. We do not want to comment further on the convergence of $u_{h}$ but refer to the paper of Molarini et al [25].

\section{Fréchet differentiability of the EIT operator}

The inverse problem of impedance tomography under the complete electrode model is to estimate the conductivity distribution $\sigma$ from all pairs of current vectors $I \in \mathbb{R}_{\diamond}^{p}$ and resulting voltage vectors $U \in \mathbb{R}_{\diamond}^{p}$. As $U$ depends linearly on $I$ for a fixed conductivity $\sigma$ there is a resistivity matrix $R \in \mathbb{R}^{p \times p}$ such that $U=R I$. This is again Ohm's law. Moreover, $R$ is symmetric for scalar real $\sigma$ [14] which we assume in the remainder of the paper. Now, we define for a fixed current vector $I$ and fixed positive contact impedances $\left(z_{j}\right)_{j=1}^{p}$

$$
\mathcal{F}: \mathcal{A} \subset L^{\infty}(B) \rightarrow H^{1}(B) \oplus \mathbb{R}_{\diamond}^{p}, \quad \sigma \mapsto(u, U),
$$

to be the forward operator that maps the conductivity $\sigma$ to the solution of the forward problem. Later we solve the inverse problem by Newton-like iterations. A necessary ingredient is the Fréchet differentiability of $\mathcal{F}$. Recall that Fréchet differentiability of $\mathcal{F}$ in $\sigma$ means that

$$
\lim _{\|\eta\|_{\infty} \rightarrow 0} \frac{\left\|\mathcal{F}(\sigma+\eta)-\mathcal{F}(\sigma)-\mathcal{F}^{\prime}(\sigma) \eta\right\|_{H^{1}(B) \oplus \mathbb{R}_{\diamond}^{p}}}{\|\eta\|_{\infty}}=0 .
$$

Theorem 4.1. Let $I$ be a fixed current vector and $z_{1}, \ldots, z_{p}$ be fixed positive contact impedances. The operator $\mathcal{F}$ which maps $\sigma \in \operatorname{int}(\mathcal{A})$ to the solution $(u, U) \in H^{1}(B) \oplus \mathbb{R}_{\diamond}^{p}$ of the forward problem with current vector I is Fréchet differentiable. If $\eta \in L^{\infty}(B)$ is such that $\sigma+\eta \in \mathcal{A}$, then the derivative $\mathcal{F}^{\prime}(\sigma) \eta=:(w, W)$ satisfies the following variational problem:

$$
-b_{\sigma}((w, W),(v, V))=\int_{B} \eta \nabla u^{0} \nabla v \mathrm{~d} x
$$

for all $(v, V) \in H^{1}(B) \oplus \mathbb{R}_{\diamond}^{p}$, where $\left(u^{0}, U^{0}\right):=\mathcal{F}(\sigma)$. 
Proof. Kaipio et al [21] give a proof in the case of the quotient space $\widetilde{H}:=\left(H^{1}(B) \oplus \mathbb{R}_{\diamond}^{p}\right) / \mathbb{R}$. However, the spaces $\widetilde{H}$ and $H^{1}(B) \oplus \mathbb{R}_{\diamond}^{p}$ are norm equivalent. Since

$$
\begin{aligned}
\|(u, U)\|_{\widetilde{H}}^{2} & =\|\nabla u\|_{L^{2}(B)}^{2}+\inf _{c \in \mathbb{R}}\left\{\|u+c\|_{L^{2}(B)}^{2}+|U+c|_{2}^{2}\right\} \\
& \leqslant\|\nabla u\|_{L^{2}(B)}^{2}+\|u\|_{L^{2}(B)}^{2}+|U|_{2}^{2}=\|(u, U)\|_{H^{1}(B) \oplus \mathbb{R}^{p}}^{2}
\end{aligned}
$$

the embedding $H^{1}(B) \oplus \mathbb{R}_{\diamond}^{p} \hookrightarrow \widetilde{H}$ is continuous and bijective. Hence, the open mapping theorem yields norm equivalence.

Theorem 4.1 shows especially that $\sigma \mapsto U$ is Fréchet differentiable as second argument of a differentiable mapping and the derivative is given by formula (4.1). The nice part of this formula is that the derivative can be computed using the variational formulation of the forward problem. On the other hand, solving this variational problem means to compute one directional derivative. Unfortunately, Newton-like methods require to compute lots of directional derivatives and this is usually the bottleneck of these algorithms.

\section{Newton-type methods for the inverse EIT problem}

In this section, we consider iterative methods of Newton-type for the inverse EIT problem. These methods work by local linearization of the nonlinear operator $\mathcal{F}$ and by regularization of the Newton step. The well-known NOSER algorithm of the Rensselaer group is one example, see Cheney et al $[12,13]$.

Assume that we apply $l$ current vectors $I^{j} \in \mathbb{R}_{\diamond}^{p}, j=1, \ldots, l$, in the EIT experiment and measure the corresponding voltage vectors $U^{j} \in \mathbb{R}_{\diamond}^{p}$. The set $\left\{I^{1}, \ldots, I^{l}\right\}$ is called a current frame. For notational convenience we define a vector

$$
I:=\left(I^{1}, \ldots, I^{l}\right)=\left(I_{1}^{1}, \ldots, I_{p}^{1}, \ldots, I_{1}^{l}, \ldots, I_{p}^{l}\right) \in \mathbb{R}^{l p},
$$

such that all $I^{j}$ 's are stored in one single column vector. Let further $U \in \mathbb{R}^{l p}$ be the column vector that arranges all the voltage vectors $U^{j}$ in the same way. For simplicity, we write $U=R I$ for $I \in \mathbb{R}^{l p}$, where $R$ is now a $l p \times l p$ matrix such that every $I^{j}$ is mapped on the corresponding $U^{j}$. For the remainder of this work we fix $I$, having in mind that we use always the same current frame.

In the next step we transform the continuous setting in a discrete one. Suppose we are given a triangulation $\mathcal{T}=\left\{T_{1}, \ldots, T_{|\mathcal{T}|}\right\}$ of the domain $B$. Then we denote by $S$ the space of step functions spanned by the indicator functions $\mathbb{1}_{T_{t}}$ and define $\mathcal{A}_{\mathrm{d}}=\mathcal{A} \cap S$, i.e., any $s \in \mathcal{A}_{\mathrm{d}}$ takes the form

$$
s(x)=\sum_{t=1}^{|\mathcal{T}|} s_{t} \mathbb{1}_{T_{t}}(x) \quad \text { for } \quad x \in B \quad \text { and } \quad s_{t}>0 .
$$

We always identify $s$ with its coordinate representation: $s=\left(s_{t}\right)_{t=1, \ldots,|\mathcal{T}|}$. Let us define the discrete forward operator $\mathcal{F}_{\mathrm{d}}$ by

$$
\mathcal{F}_{\mathrm{d}}: \mathcal{A}_{\mathrm{d}} \rightarrow \mathbb{R}^{l p}, \quad s \mapsto U=\left(R_{s} I^{1}, \ldots, R_{s} I^{l}\right) \in \mathbb{R}^{l p},
$$

where $I=\left(I^{1}, \ldots, I^{l}\right)$ is a fixed current frame in $\mathbb{R}^{l p}$ and $R_{s}$ is the resistivity matrix associated with $s \in \mathcal{A}_{\mathrm{d}}$. Note that $\mathcal{F}_{\mathrm{d}}$ can be seen as a nonlinear vector field from $\mathbb{R}^{|\mathcal{T}|} \rightarrow \mathbb{R}^{l p}$. Since 
$\mathcal{F}: \mathcal{A}_{\mathrm{d}} \rightarrow \mathbb{R}^{p}$ is Fréchet differentiable, $\mathcal{F}_{\mathrm{d}}^{\prime}$ is a matrix, called the Jacobian of $\mathcal{F}_{\mathrm{d}}$. As a consequence, if $\mathcal{F}_{\mathrm{d}}^{\prime}(s) \eta_{\mathrm{d}}=\left(W^{1}, \ldots, W^{p}\right) \in \mathbb{R}^{l p}$ then $W^{j} \in \mathbb{R}_{\diamond}^{p}$ can be computed by solving the variational problem (4.1). For the implementation of the Newton-like iterations below we need to evaluate the matrix-vector product $\mathcal{F}_{\mathrm{d}}^{\prime}(s) \eta$ and the matrix $\mathcal{F}_{\mathrm{d}}^{\prime}$. How this can be realized efficiently we explain in the appendix.

The natural norm on $S$ is a weighted Euclidean norm. For $s=\left(s_{t}\right) \in S$ we set

$$
|s|_{2, a}^{2}=\sum_{t=1}^{|\mathcal{T}|} a_{t}\left|s_{t}\right|^{2},
$$

where $a=\left(a_{t}\right)$ is the vector containing the areas of the triangles of the triangulation $\mathcal{T}$. Observe that $|s|_{2, a}=\|s\|_{L^{2}(B)}$ for any $s \in S$.

Assume now we are given measured data $U \in \mathbb{R}^{l p}$. In order to find an estimate for the corresponding conductivity distribution $\sigma$ we seek $\sigma^{\star} \in \mathcal{A}$ that fits our data $U$, that is, $\mathcal{F}\left(\sigma^{\star}\right)=U$. Note that $U$ is finite dimensional and hence there may exist lots of such $\sigma^{\star}$. In an iterative method we try to improve our actual guess $\sigma_{j} \in \mathcal{A}_{\mathrm{d}}, j \in \mathbb{N}$, by adding a correction step $h_{j}$. We wish to have $h_{j}$ such that $\sigma_{j}+h_{j}=\sigma^{\star}$. Since $\mathcal{F}_{\mathrm{d}}$ is differentiable we can write

$$
\mathcal{F}_{\mathrm{d}}^{\prime}\left(\sigma_{j}\right)\left(\sigma^{\star}-\sigma_{j}\right)=U-\mathcal{F}_{\mathrm{d}}\left(\sigma_{j}\right)-E\left(\sigma^{\star} ; \sigma_{j}\right)
$$

with the linearization error $E\left(\sigma^{\star} ; \sigma_{j}\right)$. As the linearization error is unknown we try to solve

$$
\mathcal{F}_{\mathrm{d}}^{\prime}\left(\sigma_{j}\right) h_{j} \stackrel{!}{=} U-\mathcal{F}_{\mathrm{d}}\left(\sigma_{j}\right)
$$

in the space of step functions $S$. All Newton-like solvers start in solving the above equation some way or other. Due to the ill-posedness of the inverse EIT problem [6, 7] we expect instabilities in solving (5.3). To compensate the instabilities we apply a regularization scheme to (5.3). The regularization of ill-posed problems is addressed by, e.g., Engl et al [16] or Rieder [31]. Probably the most often used approach is Tikhonov regularization where

$h_{j}=\left(\mathcal{F}_{\mathrm{d}}^{\prime \top}\left(\sigma_{j}\right) \mathcal{F}_{\mathrm{d}}^{\prime}\left(\sigma_{j}\right)+\theta_{j} \operatorname{diag}(a)\right)^{-1} \mathcal{F}_{\mathrm{d}}^{\prime \top}\left(\sigma_{j}\right)\left(U-\mathcal{F}_{\mathrm{d}}\left(\sigma_{j}\right)\right) \quad$ for $\quad \theta_{j}>0$.

Note that the perturbation term is chosen to respect the norm $|\cdot|_{2, a}$. The step $h_{j}$ serves as Newton update for our guess $\sigma_{j}$ by

$$
\sigma_{j+1}=\sigma_{j}+h_{j}
$$

The iterative scheme (5.4) and (5.5) is called the Levenberg-Marquardt method, see Lionheart and Polydorides [28] and Hanke [17]. A similar method is due to Bakushinskii [4],

$h_{j}=\left(\mathcal{F}_{\mathrm{d}}^{\prime \top}\left(\sigma_{j}\right) \mathcal{F}_{\mathrm{d}}^{\prime}\left(\sigma_{j}\right)+\theta_{j} \operatorname{diag}(a)\right)^{-1}\left(\mathcal{F}_{\mathrm{d}}^{\prime \top}\left(\sigma_{j}\right)\left(U-\mathcal{F}_{\mathrm{d}}\left(\sigma_{j}\right)\right)+\theta_{j} \operatorname{diag}(a)\left(\sigma_{0}-\sigma_{j}\right)\right)$,

where the right most term, which prevents the iterates $\sigma_{j}$ to diverge too far from the initial guess $\sigma_{0}$, brings in additional stability. The method described in equation (5.6), together with (5.5), is known as the iteratively regularized Gauß-Newton method, see, e.g., Bakushinskii and Kokurin [3] and Kaltenbacher [23, 24]. All presented regularization schemes for (5.3) are linear so far.

We have not yet mentioned how to stop the iterative schemes (5.4) or (5.6). The reason is that inverse solvers of Newton-type applied to EIT usually stop after one step, at least in the two-dimensional case. Therefore, the EIT problem is not locally but globally linearized about the initial guess $\sigma_{0}$. For the applications reported in [12, 13, 21], global linearization yields sufficient accuracy while allowing real-time reconstructions. Our numerical experiments in the next subsection are based on the following one-step solver: 

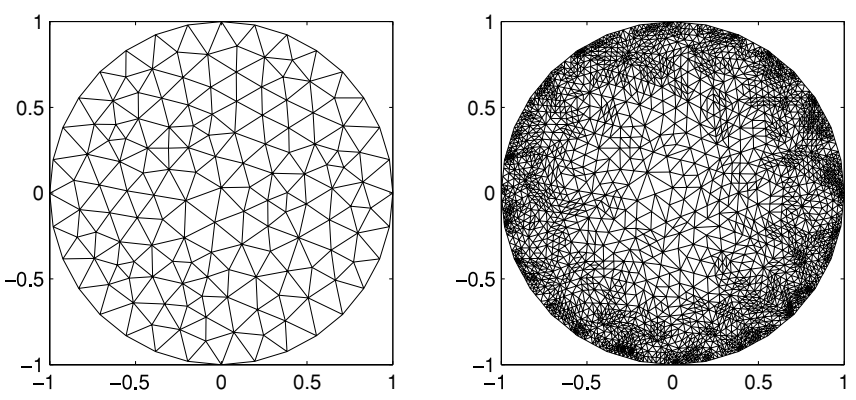

Figure 2. Left: mesh for the reconstruction when 16 electrodes are used. Right: adaptively refined mesh for the computation of the Jacobian $\mathcal{F}^{\prime}$.

Initial guess $\sigma_{0}$;

Regularization parameter $\theta$;

$\sigma_{1}=\sigma_{0}+\left(\mathcal{F}_{\mathrm{d}}^{\prime \top}\left(\sigma_{0}\right) \mathcal{F}_{\mathrm{d}}^{\prime}\left(\sigma_{0}\right)+\theta \operatorname{diag}(\mathrm{a})\right)^{-1} \mathcal{F}_{\mathrm{d}}^{\prime \top}\left(\sigma_{0}\right)\left(\mathrm{U}-\mathcal{F}_{\mathrm{d}}\left(\sigma_{0}\right)\right)$

return $\sigma_{1}$;

The NOSER ${ }^{3}$ algorithm uses the diagonal of the matrix $\mathcal{F}_{\mathrm{d}}^{\prime \top}\left(\sigma_{0}\right) \mathcal{F}_{\mathrm{d}}^{\prime}\left(\sigma_{0}\right)$ instead of the diagonal matrix $\operatorname{diag}(a)$ as in (5.4), see [13].

\subsection{Numerical experiments with NOSER-like regularization}

The experimental protocol for the numerical experiments with the Tikhonov one-step solver is the following. The data for the reconstruction algorithms are obtained synthetically. In our data retrieval we try to avoid the most obvious inverse crime and use different meshes for the forward data computation of $\mathcal{F}_{\mathrm{d}}$ and the inverse computations (i.e., the reconstruction of a conductivity). Moreover, to calculate the Newton step in (5.4) one also needs to compute the Jacobian $\mathcal{F}_{\mathrm{d}}^{\prime}$ which is defined by a variational problem and approximated using a FEM, see the appendix. For the computation of the Jacobian, a third mesh is employed. In figure 2, we show the reconstruction mesh that is used in the case of 16 electrodes together with the refined mesh to compute the Jacobian. The forward computations are done on an even more refined grid to guarantee quality of the data. The meshes for the computation of the forward operator and the Jacobian are refined towards the electrodes using the adaptive mesh refinement procedure provided by MATLABs ${ }^{4}$ partial differential equation toolbox. Of course, the computation of these meshes is performed independently of and before the inverse computations. Especially, these meshes are the same for all our examples under the same number of electrodes.

Recall that the data set for the inverse solver is the current frame $I \in \mathbb{R}^{l p}$ and the resulting voltage vector $U \in \mathbb{R}^{l p}$. In our experiments, we set $l=p$ and use current vectors of the form $(0, \ldots, 0,1,-1,0, \ldots, 0)$ which are the most simple ones and easy to implement. The question of the choice of current patterns is nontrivial and there exist concepts of optimal current patterns and distinguishability, see Isaacson [19] or Kaipio et al [22] for details. We do not care of these questions but remark that, in view of practical experiments, we only incorporate voltages from electrodes in the forward data set where no current is fed.

Newton schemes always need some initial guess as starting point for the iteration. We always use the background conductivity $\sigma \equiv 1$ as initial guess for the one-step solver (as well as later for the REGINN algorithm). This is an appropriate choice since it matches

3 Newton One-Step solvER.

4 MATLAB is a trademark of The MathWorks, Inc. 

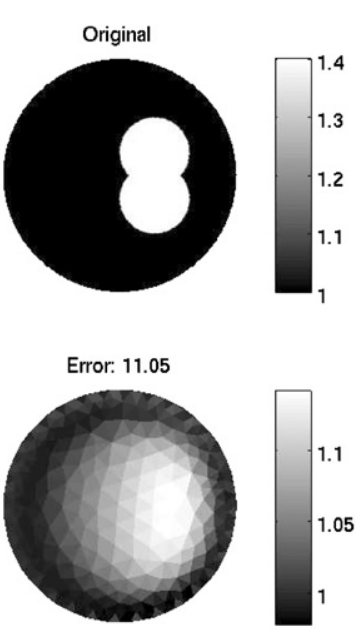
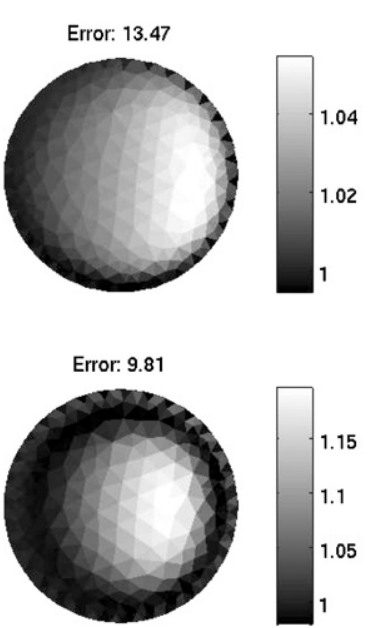

Error: 12.36
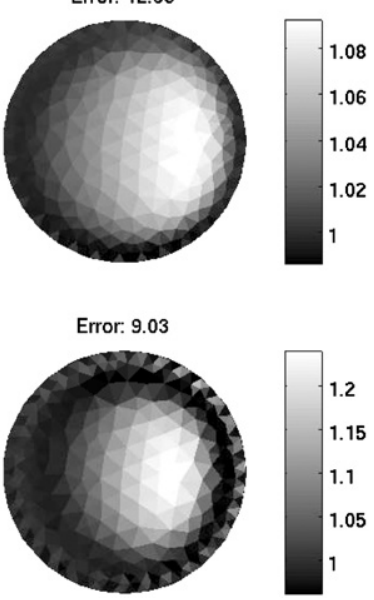

Figure 3. One-step reconstruction of a non-convex inclusion (32 electrodes, initial parameter $\theta=0.35$, no artificial noise).

the background conductivity of our examples. Recall that the complete electrode model, which is our model of choice, includes a contact impedance effect at the electrodes. In our computations, the contact impedance is set to 0.25 for all electrodes. We found this numerical value from [21, figure 4].

Some of the subsequent reconstructions are computed from synthetic data in the presence of artificial noise. Our input data for the inversion algorithm are the current patterns $I^{1}, \ldots, I^{l}$ and the corresponding voltage vectors $U^{1}, \ldots, U^{l}$ which we store for algorithmic reasons in $l \times l$-matrices. Therefore, the noise is measured in the Frobenius norm and the relative error between computed and perturbed data is given in per cent.

All figures presenting our different results are organized in the same fashion: in the upper left corner the reader finds the projection of the original conductivity distribution on a fine grid which is only used for plotting. Because of the projection the boundaries of the inhomogeneities are frayed out. Next, we plot five reconstructions where the regularization parameter $\theta$ is divided by 3 successively. All reconstructions are computed simulating an $n$-electrode system. By this term we mean a regular polygonal domain with $2 n$ corners such that every second side of the polygon is used as an electrode. We reconstruct scalar real conductivities and emphasize that the same colours (grey values) in different reconstructions do usually not refer to the same conductivity, i.e., the colourmaps of the plots are in general different.

Figure 3 shows the reconstruction of a non-convex inclusion in the form of two overlapping circles which are placed inside the domain. The reconstruction has been obtained simulating a 32-electrode system without artificial noise. We used $\theta=0.35$ as initial regularization parameter. The best reconstruction seems to be the one in the middle of the bottom row. This reconstruction shows the correct place but fails to distinguish the two circles. Nevertheless, the reconstruction seems to respect the convex hull of the non-convex inclusion. The numerical value of the conductivity of the inclusion is 1.4 and underestimated by 1.2. Moreover, the discontinuity of the inclusion is strongly smoothed by Tikhonov regularization and the electrodes close to the inclusion affect the reconstruction when the regularization parameter is small, see right plot at the bottom. Note that our reconstruction algorithm does not use penalty terms involving differential operators which might cope with this effect. Also the quasistatic 

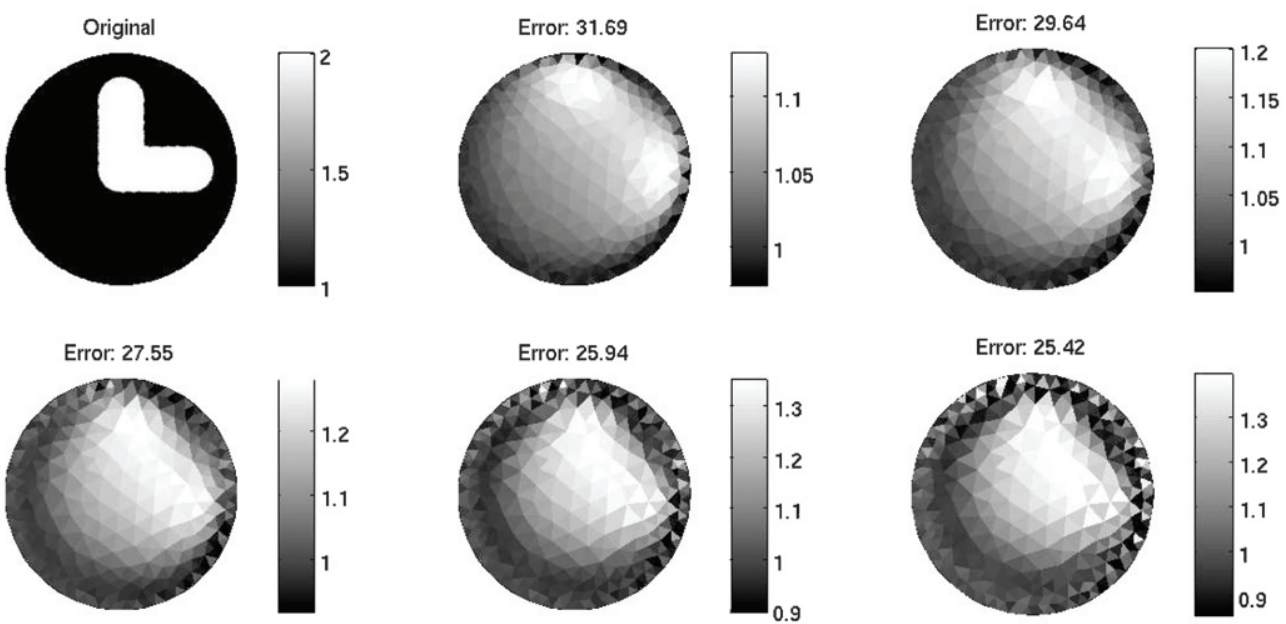

Figure 4. One-step reconstruction of an L-shaped inclusion (32 electrodes, initial parameter $\theta=0.35,0.5 \%$ artificial noise)
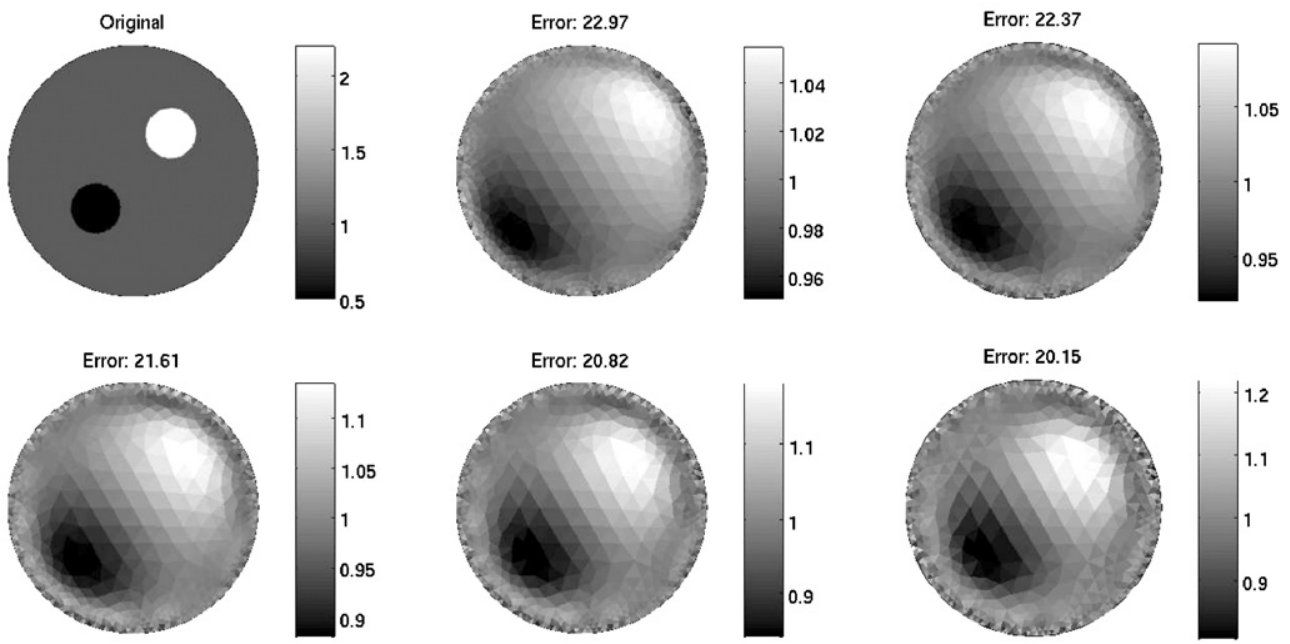

Figure 5. One-step reconstruction of two opposite inclusions (64 electrodes, initial parameter $\theta=0.48,1 \%$ artificial noise)

imaging technique [20] designed to correct errors in the electrode model does not improve the reconstructions.

Figure 4 shows reconstructions of an L-shaped inclusion. We simulated again a 32-electrode system with $0.5 \%$ artificial noise and started with $\theta=0.35$. The best reconstruction seems to be the left most of the bottom row. The location of the inclusion is found while its size is too large and the non-convexity is only slightly visible. As before, the numerical value of the conductivity is underestimated and electrodes being next to the inclusion spoil the reconstruction as the regularization parameter gets smaller.

The reconstructions up to now have been computed simulating a 32-electrode system. The plots in figure 5 are now computed simulating a 64 -electrode system. We added $1 \%$ artificial 
noise to the data and chose $\theta=0.48$ for the first reconstruction. As an additional difficulty the conductivities of the two inclusions are above and below the background conductivity. Again the locations of the two inclusions are roughly found but their sizes are overestimated. Using a small regularization parameter we are able to recover the shape satisfactorily but instabilities from the electrodes spoil the reconstruction near the boundary. On the other hand, large parameters smooth the conductivity strongly.

We have performed more numerical experiments than reported here. All our experiments showed that the Tikhonov one-step solver is able to find some main characteristics of the inclusions as, for instance, their locations. The approximate shapes can usually be guessed but complicated shapes are hard to recover, even if lots of electrodes are used. An experienced user might be able to guess the correct shape by playing with the parameters. The one-step approach offers only little control over the magnitude of the regularization and instability problems, especially near to the boundary, occur even if the information in the interior of the domain has not yet been fully exploited.

\section{The REGINN algorithm}

A very efficient iterative scheme for regularizing equation (5.3) is the method of conjugate gradients (cg-method), see, e.g., Engl et al [16, chapter 7] or Rieder [31, chapter 5.3]. It starts from an initial guess $\xi_{0} \in S$ and computes iteratively a sequence $\left(\xi_{k}\right)_{k \in \mathbb{N}}$ satisfying the minimization property

$$
\xi_{k}=\operatorname{argmin}\left\{\left|\left(U-\mathcal{F}_{\mathrm{d}}\left(\sigma_{j}\right)\right)-\mathcal{F}_{\mathrm{d}}^{\prime}\left(\sigma_{j}\right) \xi\right|_{2} \mid \xi \in S \text { and } \xi-\xi_{0} \in U_{k}\right\},
$$

where

$U_{k}=\operatorname{span}\left\{\mathcal{F}_{\mathrm{d}}^{\prime \top}\left(\sigma_{j}\right) r_{0},\left(\mathcal{F}_{\mathrm{d}}^{\prime \top}\left(\sigma_{j}\right) \mathcal{F}_{\mathrm{d}}^{\prime}\left(\sigma_{j}\right)\right) \mathcal{F}_{\mathrm{d}}^{\prime \top}\left(\sigma_{j}\right) r_{0}, \ldots,\left(\mathcal{F}_{\mathrm{d}}^{\prime \top}\left(\sigma_{j}\right) \mathcal{F}_{\mathrm{d}}^{\prime}\left(\sigma_{j}\right)\right)^{k-1} \mathcal{F}_{\mathrm{d}}^{\prime \top}\left(\sigma_{j}\right) r_{0}\right\}$,

is the $k$ th Krylov subspace with respect to the initial residual $r_{0}:=U-\mathcal{F}_{\mathrm{d}}\left(\sigma_{j}\right)-\mathcal{F}_{\mathrm{d}}^{\prime}\left(\sigma_{j}\right) \xi_{0}$. Therefore, the $k$ th iterate has the representation

$$
\xi_{k}=\xi_{0}+p_{k-1}\left[\mathcal{F}_{\mathrm{d}}^{\prime \top}\left(\sigma_{j}\right) \mathcal{F}_{\mathrm{d}}^{\prime}\left(\sigma_{j}\right)\right] \mathcal{F}_{\mathrm{d}}^{\prime \top}\left(\sigma_{j}\right) r_{0}
$$

with a suitable polynomial $p_{k-1}$ of degree $k-1$. Note that $p_{k-1}$ depends on $U-\mathcal{F}_{\mathrm{d}}\left(\sigma_{j}\right)$ making the cg-method a nonlinear regularization scheme.

In starting the cg-method with $\xi_{0}=0$ and in setting $h_{j}:=\xi_{N(j)}$ the Newton iteration (5.5) becomes

$$
\sigma_{j+1}=\sigma_{j}+p_{N(j)}\left[\mathcal{F}_{\mathrm{d}}^{\prime \top}\left(\sigma_{j}\right) \mathcal{F}_{\mathrm{d}}^{\prime}\left(\sigma_{j}\right)\right] \mathcal{F}_{\mathrm{d}}^{\prime \top}\left(\sigma_{j}\right)\left[U-\mathcal{F}_{\mathrm{d}}\left(\sigma_{j}\right)\right]
$$

where $N(j)$ is determined as the smallest number at which the relative (linear) residual is smaller than a given tolerance $\mu_{j} \in(0,1]$, that is,

$$
\left|\mathcal{F}_{\mathrm{d}}^{\prime}\left(\sigma_{j}\right) \xi_{N(j)}+\mathcal{F}_{\mathrm{d}}\left(\sigma_{j}\right)-U\right|_{2}<\mu_{j}\left|\mathcal{F}_{\mathrm{d}}\left(\sigma_{j}\right)-U\right|_{2} \leqslant\left|\mathcal{F}_{\mathrm{d}}^{\prime}\left(\sigma_{j}\right) \xi_{k}+\mathcal{F}_{\mathrm{d}}\left(\sigma_{j}\right)-U\right|_{2}
$$

for all $k=1, \ldots, N(j)-1$. A meaningful strategy to adapt $\mu_{j}$ 's dynamically is presented in (6.4).

Finally, iteration (6.3), called REGINN (REGularization based on INexact Newton iterations), has to be stopped in time to avoid noise amplification. Here, we rely on a discrepancy principle: choose $R>0$ and accept iterate $\sigma_{n}$ as approximation to the conductivity $s$ which fulfils

$$
\left|U-\mathcal{F}_{\mathrm{d}}\left(\sigma_{n}\right)\right|_{2} \leqslant R<\left|U-\mathcal{F}_{\mathrm{d}}\left(\sigma_{j}\right)\right|_{2} \quad \text { for all } \quad j=0, \ldots, n-1 .
$$


For the sake of clarity we give an algorithmic realization of REGINN in pseudo code:

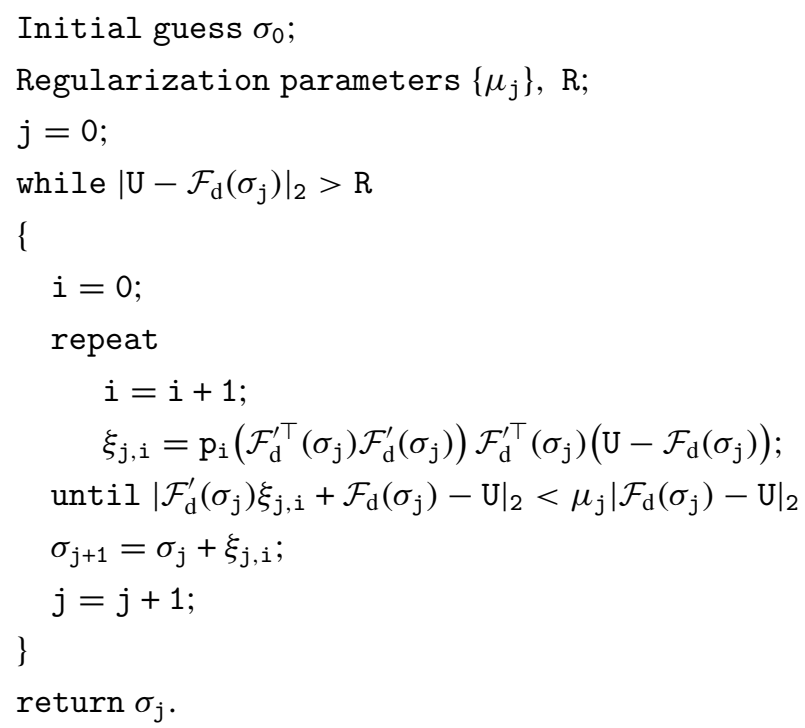

In the inner repeat-loop the Newton update is calculated using the cg-method and the outer while-loop implements the Newton iteration stopped by the discrepancy principle. REGINN was propagated by the second author and analysed in a series of papers [29, 30, 32], see also Hanke [18]. Termination of the inner and outer loops as well as stability and convergence results have been obtained for a large class of nonlinear inverse problems. At present we do not know whether the convergence analysis applies to impedance tomography as well. For instance, we do not know whether the EIT operator satisfies the tangential cone condition:

$$
\left\|\mathcal{F}(\sigma)-\mathcal{F}(\eta)-\mathcal{F}^{\prime}(\eta)(\sigma-\eta)\right\|_{H^{1}(B) \oplus \mathbb{R}_{\diamond}^{p}} \leqslant c\|\mathcal{F}(\sigma)-\mathcal{F}(\eta)\|_{H^{1}(B) \oplus \mathbb{R}_{\diamond}^{p}}
$$

locally near to the searched-for conductivity distribution for a constant $c<1$. To obtain convergence rates even stronger assumptions are required so far. Therefore, our present work is mainly experimental and numerical.

One of the big advantages of REGINN is that the tolerances $\left\{\mu_{j}\right\} \subset(0,1)$ can be adapted dynamically incorporating information on the local degree of ill-posedness gained during the iteration. The following strategy (6.4) from [29, section 6] for choosing the tolerances complies with the convergence analysis: the smaller the tolerances are the less Newton steps (passes through the while-loop) are required to terminate REGINN ([29, corollary 4.7]). On the other hand, the tolerances must not be too small to avoid noise amplification while solving (5.3) ([29, lemma 3.2 and (3.6)]). In the starting phase of REGINN the repeat-loop terminates even for small tolerances $([29,(3.6)])$. Accordingly, we start with a small tolerance and increase it during the iteration. An increase of the tolerance is needed if the number of passes through the repeat-loop of two consecutive Newton steps increases. The tolerances shall be decreased whenever successive numbers of passes through the repeat-loop drop. Moreover, we apply a safeguarding technique: if the nonlinear defect $\left|U-\mathcal{F}_{\mathrm{d}}\left(\sigma_{j}\right)\right|_{2}$ is already close to $R$, then it is unnecessary to choose a small tolerance $\mu_{j}$ since then $\left|U-\mathcal{F}_{\mathrm{d}}\left(\sigma_{j+1}\right)\right|_{2}$ might be considerably smaller than $R$.

The above considerations are realized in (6.4): initialize $\mu_{\text {start }} \in(0,1), \mu_{\max } \in\left(\mu_{\text {start }}, 1\right)$, $\zeta \in(0,1)$ and define auxiliary parameters $\tilde{\mu}_{0}=\tilde{\mu}_{1}=\mu_{\text {start }}$. Then,

$\mu_{j}:=\mu_{\max } \max \left\{R /\left|U-\mathcal{F}_{\mathrm{d}}\left(\sigma_{j}\right)\right|_{2}, \tilde{\mu}_{j}\right\}, \quad j=0,1, \ldots, N(j)-1$, 
Table 1. Parameter adaption of REGINN during the reconstruction process. The corresponding reconstruction is shown in figure 6. Entries in column $j$ denote the outer iteration counter, $N(j)$ is the number of inner iterations in the $j$ th step and $\mu_{j}$ is the chosen tolerance for the $j$ th step. The relative error is given in per cent.

\begin{tabular}{rlll}
\hline$j$ & $N(j)$ & $\mu_{j}$ & Error \\
\hline 0 & 0 & - & 34.72 \\
1 & 2 & 0.799 & 29.92 \\
2 & 4 & 0.799 & 27.35 \\
3 & 3 & 0.899 & 27.20 \\
4 & 6 & 0.871 & 25.88 \\
5 & 2 & 0.935 & 25.84 \\
6 & 6 & 0.906 & 25.01 \\
7 & 1 & 0.968 & 25.00 \\
8 & 6 & 0.938 & 24.52 \\
9 & 1 & 0.989 & 24.52 \\
10 & 2 & 0.958 & 24.50 \\
11 & 1 & 0.978 & 24.50 \\
12 & 5 & 0.948 & 24.30 \\
13 & 1 & 0.989 & 24.29 \\
14 & 5 & 0.958 & 24.15 \\
15 & 1 & 0.991 & 24.15 \\
\hline
\end{tabular}

where

$$
\tilde{\mu}_{j}:= \begin{cases}1-\frac{N(j-2)}{N(j-1)}\left(1-\mu_{j-1}\right): & N(j-1) \geqslant N(j-2), \quad j \geqslant 2 . \\ \zeta \mu_{j-1}: & \text { otherwise, }\end{cases}
$$

In our numerical experiments for impedance tomography we worked with the parameter set-up $\mu_{\text {start }}=0.8, \mu_{\max }=0.99$ and $\zeta=0.97$.

\subsection{Numerical experiments with the REGINN algorithm}

The experimental protocol for the experiments with the REGINN algorithm is the same as for the Tikhonov one-step solver in section 5.1. More precisely, we compute the synthetical data and the Jacobian of the forward operator with the complete model on two different meshes which are both very fine near the electrodes, whereas the inverse solver works on a coarse mesh.

In experimenting with REGINN, the residual error $\left|U-\mathcal{F}_{\mathrm{d}}\left(\sigma_{j}\right)\right|_{2}$ of the iterates $\sigma_{j}$ does sometimes increase during the outer iteration process. Usually, this happens when the iteration reaches the saturation point. Possible interpretations are twofold. On one hand, the regularization parameter $R$ could be too small. On the other hand, the residual error may not decrease monotonically for the EIT problem since EIT does not belong to the class of problems where we can prove monotone decrease. Our implementation solves this problem from the numerical point of view. If the residual error increases, then we have no hope that the error itself decreases and we stop the iteration.

With the initial tolerance $\mu_{0}=0.8$, we found that the tolerances increase round about monotonically during the reconstruction process and therefore we believe that these values are adapted to the problem (compare table 1). When we perturb the data with artificial noise we measure the perturbation of the data in the Frobenius norm as mentioned in section 5.1 and indicate the relative error between computed and perturbed data in per cent. As for the 


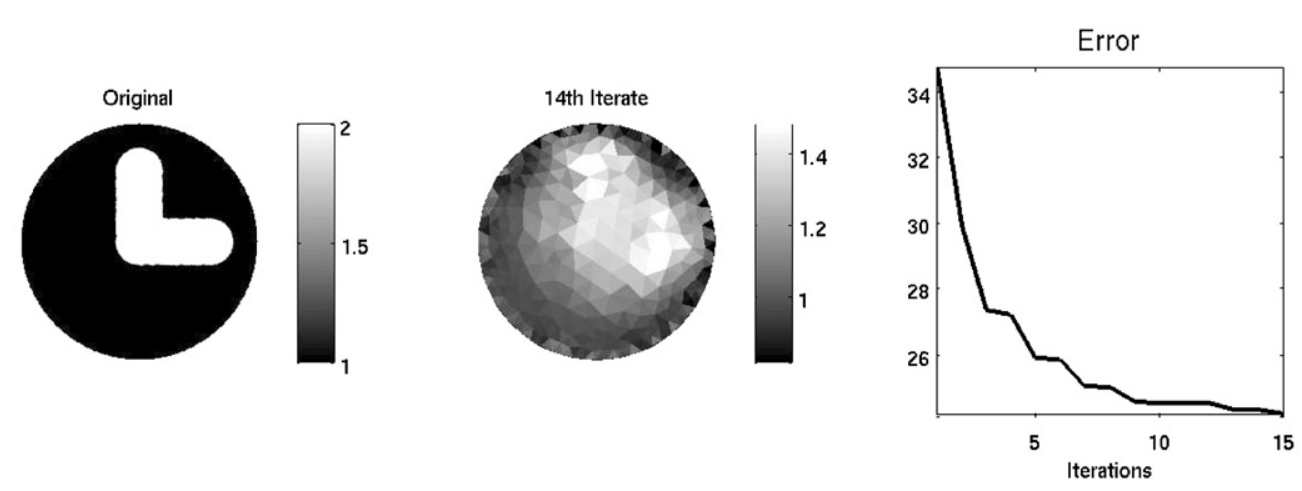

Figure 6. REGINN reconstruction of an L-shaped inclusion ( 32 electrodes, $R=1, \mu_{0}=0.8,0.5 \%$ artificial noise).

one-step solver, we always use a constant conductivity (with value 1) as initial guess for the inverse solver.

The REGINN reconstructions are presented together with the original conductivity and the evolution of the relative error during the (outer) iteration. In figure 6, we observe that REGINN is able to find the approximate shape of the L-shaped inclusion from figure 4 . We used 32 electrodes in this example and added $0.5 \%$ artificial noise. The size of the reconstructed inclusion is moderately larger than the original. Only little noise comes from the electrodes and the convex corners of the inclusion are quite well determined compared to figure 4.

The stability of the REGINN reconstructions is controlled by the adaptively chosen tolerances $\mu_{j}$. Table 1 shows this adaption process for the example of figure 6 . The tolerance selection scheme (6.4) works as predicted: For instance, from step 3 to step 4 the number of inner iterations increases from 3 to 6 and REGINN accordingly chooses $\mu_{5}$ larger than $\mu_{4}$. On the other hand, the number of inner iterations drops from 6 to 2 from step 4 to step 5 and REGINN selects a $\mu_{6}$ smaller than $\mu_{5}$. During the complete iteration process the tolerances increase from 0.799 to 0.991 .

The conductivity distribution in figure 7 is the same as in figure 5 as are the number of electrodes ( $p=64$ ) and the noise level (1\%). REGINN locates the inclusions correctly and also shows that their conductivities are above and below the reference conductivity. The REGINN reconstructions are more concentrated and less smoothed than the NOSER-like reconstructions in figure 5 .

The searched-for conductivity of figure 8 is smooth and attains values above and below the background medium. The reconstruction is computed with simulated data of a 64-electrode system with $1 \%$ artificial noise and parameters $R=2.5, \mu_{0}=0.8$. The location of the two inhomogeneities is found, their size is slightly overestimated.

In figure 9, we investigate the reconstruction of a jump conductivity distribution being not an inclusion in a homogeneous background medium, that is, we have a discontinuity also on the boundary of the domain. We corrupted the 64 electrodes data by $2 \%$ artificial noise and chose $R=3.6$ and $\mu_{0}=0.8$. REGINN finds the boundary between the two constant parts of the conductivity accurately. The two values of the conductivity are well approximated in both parts of the domain, however, in the upper half of the domain the electrodes are clearly visible.

Figure 10 presents the reconstruction of two close circular discs that are placed close to the boundary of the domain. We worked with 64 electrodes, $5 \%$ artificial noise and chose $\mu_{0}=0.8$ 

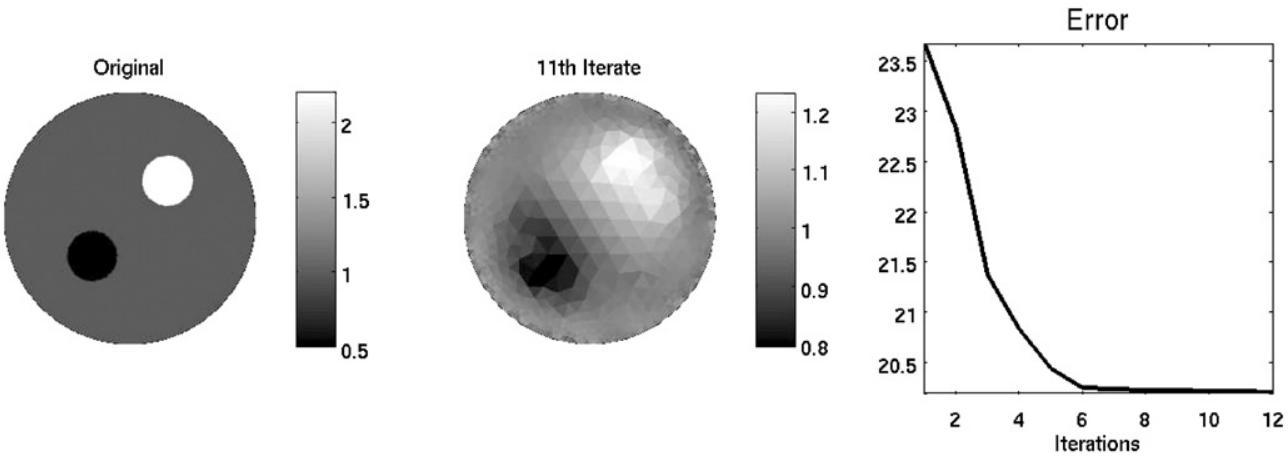

Figure 7. REGINN reconstruction of two circles with different conductivities (64 electrodes, $R=2.5, \mu_{0}=0.8,1 \%$ artificial noise).
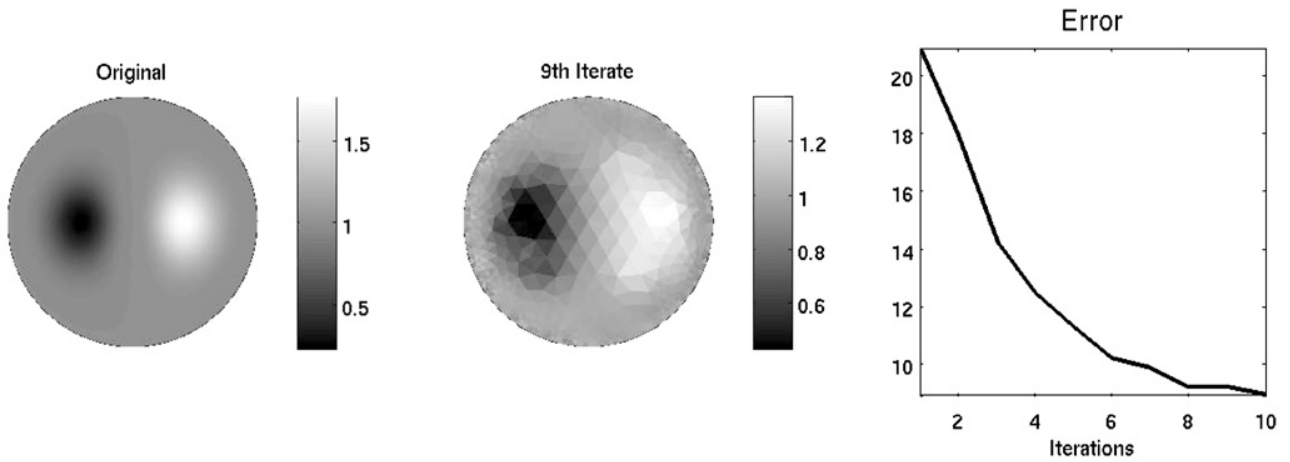

Figure 8. REGINN reconstruction of two smooth inclusions (64 electrodes, $R=2.5, \mu_{0}=$ $0.8,1 \%$ artificial noise).
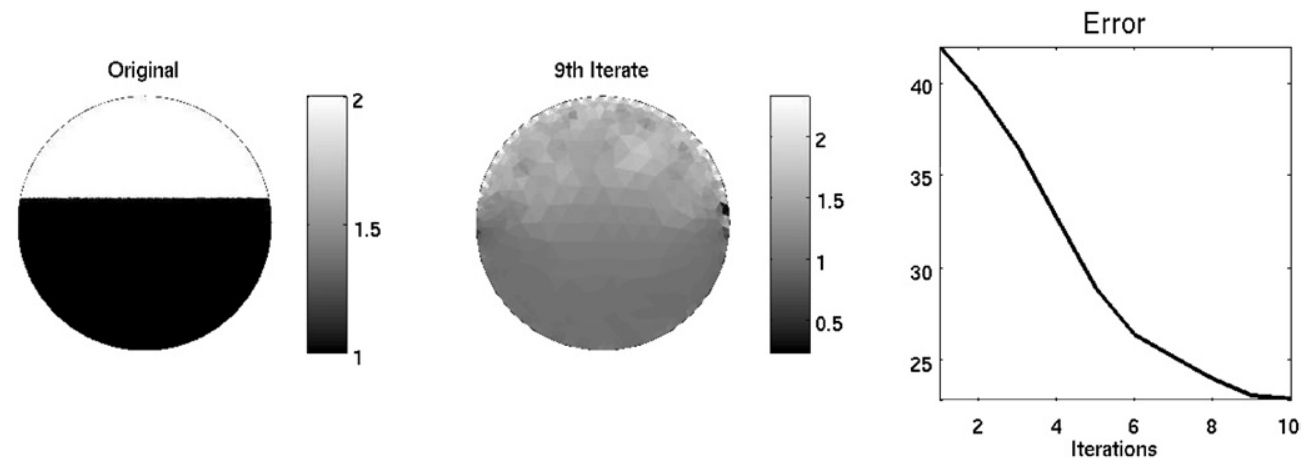

Figure 9. REGINN reconstruction of piecewise constant conductivity (64 electrodes, $R=$ 3.6, $\mu_{0}=0.8,2 \%$ artificial noise).

and $R=8$. The two inclusions are located but the numerical value of the conductivity is strongly underestimated. REGINN fails to reconstruct the two separated discs but indicates at least slightly the non-convexity of the inclusion. Noise appears near to the boundary of the domain but this is to be expected under a noise level of $5 \%$. 


\begin{tabular}{|c|c|c|c|c|c|c|c|c|}
\hline \multirow[t]{14}{*}{ Original } & \multirow{6}{*}{\multicolumn{8}{|c|}{$\begin{array}{l}\text { Figure 10. REGINN reconstruction of two close circles ( } 64 \text { electrodes, } \\
\text { artificial noise). } \\
\text { Table 2. Relative errors in per cent for different conductivity distributions } \\
\text { artificial noise, } R=1.5 \text { ). Computing time } t \text { of the algorithms is given in } \\
\text { number of outer iterations that REGINN performed. }\end{array}$}} \\
\hline & & & & & & & & \\
\hline & & & & & & & & \\
\hline & & & & & & & & \\
\hline & & & & & & & & \\
\hline & & & & & & & & \\
\hline & \multirow[b]{2}{*}{ Figure } & \multicolumn{3}{|c|}{ REGINN } & \multicolumn{4}{|c|}{ Tikhonov } \\
\hline & & $\mu_{0}=0.8$ & $t$ & $H$ & $\theta=0.04$ & $t$ & $\theta=0.13$ & $t$ \\
\hline & 3 & 10.3 & 8.2 & 2 & 11.0 & 3.2 & 9.8 & 3.3 \\
\hline & 4 & 24.1 & 45.4 & 15 & 27.5 & 3.2 & 25.9 & 3.3 \\
\hline & 5 & 20.0 & 8.4 & 2 & 20.4 & 3.3 & 19.8 & 3.2 \\
\hline & 9 & 23.9 & 27.8 & 9 & 29.3 & 3.5 & 32.4 & 3.4 \\
\hline & 10 & 21.6 & 36.2 & 12 & 21.6 & 3.2 & 20.7 & 3.6 \\
\hline & 8 & 12.0 & 66.7 & 23 & 14.5 & 3.2 & 12.5 & 3.2 \\
\hline
\end{tabular}

REGINN applied to the inverse EIT problem takes full advantage of the dynamic adaption of the level of regularization to the local degree of ill-posedness. Therefore, REGINN outperforms the Tikhonov one-step method in general with respect to image quality since less noise ease the interpretation of the reconstructions, especially near to the boundary. The images are more focused and have a higher contrast.

\section{Quantitative comparison}

In the last section we compared the classical Tikhonov one-step solver with REGINN in a qualitative way and found that REGINN produces reconstructions of at least the same quality as Tikhonov one-step reconstruction while avoiding some of its drawbacks. Now we will substantiate this observation with a quantitative study. We consider the relative error of both reconstruction methods with respect to the original resistivity distribution in the discrete $L^{2}(B)$ norm (5.2). Note that our results presented in this section are stable under small changes in the parameters $\mu_{0}, R$ and $\theta$ of the algorithms.

Table 2 compares the inverse solvers for a 32-electrode system and $0.5 \%$ artificial noise. We see that REGINN produces smaller or comparable errors than Tikhonov regularization, but does never perform significantly worse. This observation agrees with our experience and with our examples presented in sections 5 and 6 . Our computations have been carried out under Matlab 7 (R2006a) on an AMD Athlon 3800+ processor. The measured computing time 
Table 3. Relative errors in per cent for different resistivity distributions ( 64 electrodes, $1 \%$ artificial noise, $R=2.5$ ).

\begin{tabular}{rlll}
\hline & REGINN & \multicolumn{2}{c}{ Tikhonov } \\
\cline { 3 - 4 } Figure & $\mu_{0}=0.8$ & $\theta=0.053$ & $\theta=0.017$ \\
\hline 3 & 9.59 & 10.95 & 9.95 \\
4 & 22.6 & 27.5 & 26.1 \\
5 & 20.2 & 21.6 & 20.8 \\
9 & 22.8 & 31.1 & 33.8 \\
10 & 18.6 & 21.8 & 21.1 \\
8 & 8.91 & 14.3 & 12.7 \\
\hline
\end{tabular}

Table 4. Relative errors in per cent for different resistivity distributions (64 electrodes, $5 \%$ artificial noise, $R=8$ ).

\begin{tabular}{clll}
\hline & REGINN & \multicolumn{2}{c}{ Tikhonov } \\
\cline { 3 - 4 } Figure & $\mu_{0}=0.8$ & $\theta=0.078$ & $\theta=0.025$ \\
\hline 3 & 9.74 & 11.9 & 11.6 \\
4 & 25.2 & 28.4 & 27.0 \\
5 & 20.0 & 22.1 & 21.7 \\
9 & 22.3 & 30.5 & 32.9 \\
10 & 19.8 & 22.4 & 21.9 \\
8 & 10.2 & 15.3 & 14.4 \\
\hline
\end{tabular}

excludes data preprocessing or plotting. The computing time of the Jacobian is included in the case of the one-step solver, although the Jacobian could be assembled in advance. REGINN's computing time is seen to be roughly the computing time of the one-step solver times the number of outer iterations. This observation remains true when increasing the number of electrodes. Therefore, we omit CPU times in tables 3 and 4. For the present experiment (32 electrodes and a low noise level), the higher quality of REGINN's reconstructions, as discussed in section 6 , is paid for by a higher computing time. Nevertheless, REGINN is worth its price as the one-step solver is clearly outperformed under a larger number of electrodes and/or stronger noise.

Table 3 contains results for a 64 -electrode system with $1 \%$ artificial noise. REGINN now outperforms the Tikhonov one-step solver as the differences between the two algorithms are more pronounced than for the 32-electrode system. This indicates, as we think, that REGINN extracts more information from perturbed data, compare, e.g., the performance of REGINN when reconstructing the conductivity of figure 9 .

Finally, we confirm our observations once again: table 4 compares the two algorithms for a 64-electrode system under 5\% relative noise.

Let us summarize our experimental findings for the inverse EIT problem: compared to the simple Tikhonov one-step solver REGINN is able to extract more structural information from noisy data. Additionally, it profits more strongly from increasing the number of electrodes. All these advantages of REGINN originate, as we think, from the adaptive choice of the tolerances (6.4) allowing a fine-tuned regularization of the locally linearized problems (5.3).

Of course, the Tikhonov one-step solver has its advantages: only one linear problem has to be solved (if the regularization parameter $\theta$ is determined a priori!) making it a relatively fast algorithm. It is therefore hard to beat if the measured data contain little information on the searched-for conductivity as in the case of few electrodes. 
Nevertheless, our experiments reveal REGINN to be a competitive solver for the inverse EIT problem. Its proven potential deserves further exploration under real-life conditions.

\section{Appendix. Computing the Jacobian}

In this appendix, we explain how to compute the Jacobian $\mathcal{F}_{\mathrm{d}}^{\prime}(s)$ efficiently as we learned from [28]. Let us denote by $\nabla_{t} \mathcal{F}_{\mathrm{d}}(s)$ the gradient of $\mathcal{F}_{\mathrm{d}}(s)$ with respect to the $t$ th component of $s \in \mathcal{A}_{\mathrm{d}}$. Then $\mathcal{F}_{\mathrm{d}}^{\prime}$ has the form

$$
\mathcal{F}_{\mathrm{d}}^{\prime}(s)=\left(\begin{array}{c}
\nabla_{1} \mathcal{F}_{\mathrm{d}}(s)^{\top} \\
\nabla_{2} \mathcal{F}_{\mathrm{d}}(s)^{\top} \\
\vdots \\
\nabla_{|\mathcal{T}|} \mathcal{F}_{\mathrm{d}}(s)^{\top}
\end{array}\right) \in \mathbb{R}^{|\mathcal{T}| \times l p} .
$$

Recall that theorem 4.1 shows how to compute the partial derivatives appearing in (A.1). If we denote

$$
\nabla_{t} \mathcal{F}_{\mathrm{d}}(s)=: W_{t}=\left(W_{t}^{1}, \ldots, W_{t}^{l}\right) \in \mathbb{R}^{l p},
$$

then we can compute the vector $W_{t}^{m} \in \mathbb{R}_{\diamond}^{p}$ as part of the solution $\left(w_{t}^{m}, W_{t}^{m}\right) \in H^{1}(B) \oplus \mathbb{R}_{\diamond}^{p}$ of the variational problem

$$
b_{s}\left(\left(w_{t}^{m}, W_{t}^{m}\right),(v, V)\right)=-\int_{B} \mathbb{1}_{T_{t}} \nabla u^{m} \nabla v \mathrm{~d} x
$$

for all $(v, V) \in H^{1}(B) \oplus \mathbb{R}_{\diamond}^{p}$, where $u^{m} \in H^{1}(B)$ is the solution of the forward problem with respect to the current pattern $I^{m}$ and the contact impedance vector $z$, see theorem 4.1.

The reader might feel that this way of computing the Jacobian is highly expensive. One computes indeed not only the needed vectors $W_{1}, \ldots, W_{|\mathcal{T}|+p}$ but also all the potentials $w^{m} \in H^{1}(B)$ which are not needed a priori. In fact, using this method, one has to compute $l \cdot|\mathcal{T}|$ forward problems.

Fortunately, we are able to simplify substantially the computation of the Jacobian by the following trick: we introduce the auxiliary 'current frame' $J=\left(J^{1}, \ldots, J^{p}\right)$, with $J^{k}$ being the Kronecker symbol

$$
J^{k}:=\left(\delta_{j, k}\right)_{j=1}^{p} \quad \text { for } \quad k=1, \ldots, p,
$$

and let $\left(v^{k}, V^{k}\right) \in H^{1} \oplus \mathbb{R}_{\diamond}^{p}$ be the (grounded) solution of the variational problem

$$
b_{s}\left(\left(v^{k}, V^{k}\right),(y, Y)\right)=\left\langle J^{m}, Y\right\rangle=Y_{m} \quad \text { for all } \quad(y, Y) \in H^{1}(B) \oplus \mathbb{R}_{\diamond}^{p}
$$

for $k=1, \ldots, p$. Even if $J^{k}$ are no current patterns in the usual sense $\left(\sum_{j} J_{j}^{k} \neq 0\right)$, this problem is well-posed, because the linear form on the right-hand side is bounded and the bilinear form on the left is an elliptic form on $H^{1} \oplus \mathbb{R}_{\diamond}^{p}$ [14]. Then we compute

$$
\begin{aligned}
\nabla_{t} F(s) & =\left(W_{t}^{1}, \ldots, W_{t}^{l}\right)=\left(\left\langle W_{t}^{m}, J^{k}\right\rangle_{k=1}^{p}\right)_{m=1}^{l} \\
& =\left(b_{s}\left(\left(v^{k}, V^{k}\right),\left(w_{t}^{m}, W_{t}^{m}\right)\right)\right)_{l, m} \\
& \stackrel{(A .2)}{=}\left(\int_{B} \mathbb{1}_{T_{t}} \nabla u_{t}^{m} \nabla v^{k} \mathrm{~d} x\right)_{l, m} \\
& =\left(\int_{T_{t}} \nabla u_{t}^{m} \nabla v^{k} \mathrm{~d} x\right)_{l, m} .
\end{aligned}
$$

Hence, all we have to do to obtain the Jacobian is to compute the $p$ forward problems for $\left(v^{k}, V^{k}\right)$, the $l$ forward problems for $\left(u^{m}, U^{m}\right)$ and to assemble the obtained solution in the 
way indicated by (A.3). This requires the solution of $p+l$ forward problems. As $p \ll|\mathcal{T}|$ in general, the reduction of the numerical effort is tremendous. Moreover, the computation of the forward solutions always uses the same bilinear form and this fact provides additional speedup for the implementation.

An experienced reader might object to computing the Jacobian at all: REGINN, as an iterative solver, only requires the action of the Jacobian and its adjoint on a vector. Both matrix-vector products can indeed be realized by solving variational problems, see (4.1) for $\mathcal{F}_{\mathrm{d}}^{\prime}(\sigma) \eta$. However, observe the appearance of $\mathcal{F}(\sigma)$ on the right-hand side of (4.1). To set up the right-hand side for computing $\mathcal{F}_{\mathrm{d}}^{\prime}(\sigma) \eta$, one accordingly needs to evaluate the forward operator $\mathcal{F}_{\mathrm{d}}$ which means solving an additional elliptic problem. Moreover, (4.1) addresses the case of one single current vector only. Our setting deals with current frames of size $l$. So we need to solve $2 l$ elliptic problems all in all to evaluate $\mathcal{F}_{\mathrm{d}}^{\prime}(\sigma) \eta$ (neglecting the structural work for setting up the right-hand side). Since we work with $p=l$ in our implementations, the evaluation of $\mathcal{F}_{\mathrm{d}}^{\prime}(\sigma) \eta$ requires to solve $2 p$ forward problems. The same numerical effort is needed to obtain $\mathcal{F}_{\mathrm{d}}^{\prime}(\sigma)^{\top} \zeta$ via variational problems. Computing the Jacobian explicitly is therefore the cheaper way.

\section{References}

[1] Astala K and Päivärinta L 2006 A boundary integral equation for Calderón's inverse conductivity problem Proc. 7th Int. Conf. on Harmonic Analysis and Partial Differential Equations (EI Escorial, Spain, June 2004) Collect. Math. at press

[2] Astala K and Päivärinta L 2006 Calderón's inverse conductivity problem in the plane Ann. Math. 163 265-99

[3] Bakushinskii A B and Kokurin M Y 2004 Iterative Methods for Approximate Solution of Inverse Problems (Mathematics and its Applications vol 577) (Dordrecht: Springer)

[4] Bakushinskii A B 1992 The problem of the convergence of the iteratively regularized Gauss-Newton method Comput. Maths. Math. Phys. 32 1353-9

[5] Borcea L 2001 A nonlinear multigrid for imaging electrical conductivity and permittivity at low frequency Inverse Problems 17 329-59

[6] Borcea L 2002 Electrical impedance tomography Inverse Problems 18 R99-136

[7] Brühl M 1999 Gebietserkennung in der elektrischen Impedanztomographie $P h D$ Thesis Universität Karlsruhe

[8] Brühl M and Hanke M 2000 Numerical implementation of two noniterative methods for locating inclusions by impedance tomography Inverse Problems 16 1029-42

[9] Brühl M and Hanke M 2003 Recent progress in electrical impedance tomography Inverse Problems 19 S65-90

[10] Brühl M, Hanke M and Pidcock M 2001 Crack detection using electrostatic measurements Math. Model. Numer. Anal. 35 595-605

[11] Caldéron A 1980 On an inverse boundary value problem Seminar of Numerical Analysis and Its Applications to Continuum Physics (Rio de Janeiro: Soc. Brasileira de Mathemătica) pp 65-73 (online available from www.ma.umist.ac.uk/bl/Calderon)

[12] Cheney M, Isaacson D and Newell J 1999 Electrical impedance tomography SIAM Rev. 41 85-101

[13] Cheney M, Isaacson D, Newell J, Simske S and Goble J 1990 Noser: an algorithm for solving the inverse conductivity problem Int. J. Imag. Syst. Technol. 2 66-75

[14] Cheney M, Isaacson D and Somersalo E 1992 Existence and uniqueness for electrode models for electric current computed tomography SIAM J. Appl. Math. 52 1023-40

[15] Cheng K-S, Isaacson D, Newell J and Gisser D 1989 Electrode models for electric current computed tomography IEEE Trans. Biomed. Eng. 36 918-24

[16] Engl H W, Hanke M and Neubauer A 1996 Regularization of Inverse Problems (Dordrecht: Kluwer)

[17] Hanke M 1997 A regularizing Levenberg-Marquardt scheme, with applications to inverse groundwater filtration problems Inverse Problems 13 79-95

[18] Hanke M 1998 Regularizing properties of a truncated Newton-CG algorithm for nonlinear inverse problems Numer. Funct. Anal. Optim. 18 971-93

[19] Isaacson D 1986 Distinguishability of conductivities by electric current computed tomography IEEE Trans. Med. Imaging 5 91-5

[20] Järvenpäa S and Somersalo E 1997 Impedance imaging and electrode models Inverse Problems in Medical Imaging and Nondestructive Testing (Oberwolfach Conf., 1996) ed H Engl, A Louis and W Rundell pp 65-74 
[21] Kaipio J, Kolehmainen V, Somersalo E and Vaukhonen M 2000 Statistical inversion and Monte Carlo sampling methods in electrical impedance tomography Inverse Problems 16 1487-522

[22] Kaipio J and Somersalo E 2004 Statistical and Computational Inverse Problems (Berlin: Springer)

[23] Kaltenbacher B 1997 Some Newton-type methods for the regularization of nonlinear ill-posed problems Inverse Problems 13 729-53

[24] Kaltenbacher B 1998 A posteriori parameter choice strategies for some Newton type methods for the regularization of nonlinear ill-posed problems Numer. Math. 79 501-28

[25] Molinari M, Cox S, Blott B and Daniell G 2001 Adaptive mesh refinement techniques for electrical impedance tomography Physiol. Meas. 22 91-6

[26] Mueller J L and Siltanen S 2003 Direct reconstructions of conductivities from boundary measurements SIAM J. Sci. Comput. 24 1232-66

[27] Nachman A 1995 Global uniqueness for a two-dimensional inverse boundary value problem Ann. Math. 142 71-96

[28] Polydorides N and Lionheart W 2002 A Matlab toolkit for three-dimensional impedance tomography Meas. Sci. Technol. 13 1871-83

[29] Rieder A 1999 On the regularization of nonlinear ill-posed problems via inexact Newton iterations Inverse Problems 15 309-27

[30] Rieder A 2001 On convergence rates of inexact Newton regularizations Numer. Math. 88 347-65

[31] Rieder A 2003 Keine Probleme mit Inversen Problemen (Wiesbaden: Vieweg)

[32] Rieder A 2005 Inexact Newton regularization using conjugate gradients as inner iteration SIAM J. Numer. Anal. 43 604-22

[33] Siltanen S, Mueller J and Isaacson D 2000 An implementation of the reconstruction algorithm of A. Nachman for the 2D inverse conductivity problem Inverse Problems 16 681-99

[34] Siltanen S, Mueller J and Isaacson D 2001 An implementation of the reconstruction algorithm of A. Nachman for the 2D inverse conductivity problem Inverse Problems 17 1561-3 (Erratum)

[35] Sylvester J and Uhlmann G 1986 A uniqueness theorem for an inverse boundary value problem in electrical prospection Commun. Pure Appl. Math. 39 92-112

[36] Sylvester J and Uhlmann G 1987 A global uniqueness theorem for an inverse boundary value problem Ann. Math . 125 69-153

[37] Sylvester J and Uhlmann G 1988 Inverse boundary value problems at the boundary-continuous dependence Commun. Pure Appl. Math. 41 197-219 